\title{
Editorial comments to the articles published in Issues 1-3 of Volume 6
}

\author{
Hiroshi Miyamoto ${ }^{1,2,3 *}$ \\ ${ }^{1}$ Department of Pathology \& Laboratory Medicine, University of Rochester Medical Center, USA \\ ${ }^{2}$ Department of Urology, University of Rochester Medical Center, USA \\ ${ }^{3}$ James P. Wilmot Cancer Institute, University of Rochester Medical Center, USA
}

In Issues 1-3 of Volume 6, six original Research Articles and four Case Reports, in addition to Editorials and Commentaries, were published.

Guben Yiliu II (GY II), a mixture of extracts from 13 species of herbs, has been prescribed in China as traditional Chinese medicine. Bai et al. [1] determined anti-tumor activity of GY II, using cell line models for laryngeal cancer. GY II was found to considerably inhibit the cell viability of the Hep-2 line and primary culture from patients and induce G0/G1 cell-cycle arrest as well as apoptosis. GY II also inhibited cell migration and invasion. These observations supported anti-tumor property of traditional Chinese medicine.

The precise nature of the Warburg effect, where it was assumed almost a century ago that cancer cells produce their energy via a high rate of glycolysis and subsequent fermentation of glucose to lactate, remains unclear. Pomuceno-Orduñez et al. [2] assessed the consequence of periodic glucose deprivation, using a recently proposed mathematical model with modifications, which was found to increase the complexity of glycolytic oscillations and lower the cellular energy charge in tumor cells. These findings may provide a strategy to enhance the efficacy of anti-tumor therapy.

A cross-sectional study by Amamou et al. [3], involving 138 adult patients diagnosed with cancer and their family caregivers, assessed the impact of demographic, clinical, and psychological variables on the perception of burden. The authors reported high levels of burden from caregiving, particularly in those who were males, at age of 40-59 years, full-time employees, or children of the patients, as well as those who had another family member that required daily care, cared for more than one year, or did not seek help from a healthcare professional at home, emphasizing the importance of systematic assessment and early intervention.

Blebs can be formed through invasive migration of cancer cells. Ponuwei and Dash [4] investigated the role of aquaporins (AQPs), integral membrane proteins that form pores in the cell membranes, in bleb formation in cancer cells. Using cell lines cultured in 3D Matrigel matrix, the authors demonstrated that knockdown of AQP1 via siRNA resulted in considerable inhibition of bleb formation, whereas overexpression of AQP1 significantly increased the size of blebs as well as the speed of bleb retraction for which activation of $\mathrm{Na}^{+} / \mathrm{H}^{+}$exchange was required. AQP1 may thus offer a therapeutic target for preventing cancer cell migration.

Physical activity has been known to contribute to considerably reducing the risk of cancer development and improving healthy survival of cancer patients. A pilot study by Magro et al. [5] aimed to assess the impact of physical activity on the anthropometric and physical parameters that could particularly predict the risks of cardiovascular disease. This study indeed verified that an exercise prescription program recommended by the World Cancer Research Fund could significantly improve body mass index/body weight and waist or hip circumference in female breast cancer survivors.

Anastomotic leakage is a major complication of upper gastrointestinal surgery, which is often required for second surgery. In a study by Makino et al. [6], minimally invasive endoscopic management for leakage or fistula of anastomosis following esophagectomy or gastrectomy was introduced. These procedures included clipping and fibrin glue injection, Over-The-Scope Clip placement, and stent insertion. Based on their experience, the authors proposed a diagram showing adequate management in patients with post-esophagectomy/ gastrectomy leak. In particular, its size appeared to be one of the most critical factors for management selection.

\section{References}

1. Bai Y, Wang R, Cui X, Feng L, Shi Q, et al. (2019) GY II represses proliferation and metastasis and promotes apoptosis in laryngeal squamous cell carcinoma. Integr Cancer Sci Ther 6: 1000298.

2. Pomuceno-Orduñez JP, Silva E, Martin RR, Durán I, Bizzarri M, et al. (2019) Phase transitions in tumor growth VII: The effect of periodic glucose pulses and privations in a cancer model. Integr Cancer Sci Ther 6: 1000301.

3. Amamou B, Fathallah S, Ammar D, Mhalla A, Zaafrane F, et al. (2019) Impact of demographic, clinical and psychological variables of patients and caregivers on the perception of burden among the family caregivers of patients with cancer. Integr Cancer Sci Ther 6: 1000303.

4. Ponuwei GA, Dash PR (2019) Aquaporin 1 (AQP1) regulates plasma membrane bleb formation by facilitating bleb retraction phase in cancer cells. Integr Cancer Sci Ther 6: 1000305 .

*Correspondence to: Hiroshi Miyamoto, $\mathrm{MD}, \mathrm{PhD}$, Department of Pathology \& Laboratory Medicine, University of Rochester Medical Center, 601 Elmwood Avenue, Box 626, Rochester, NY 14642, USA, Tel: +1(585)275-8748, E-mail: hiroshi_miyamoto@urmc.rochester.edu

Received: June 03, 2019; Accepted: June 20, 2019; Published: June 24, 2019 
5. Magro S, Sofi F, Mascherini G, Galanti G, Stefani L (2019) Concordance between the WCRF recommendations and reduced global cardiovascular risk in a cohort of survived breast cancer patients. Integr Cancer Sci Ther 6: 1000306.
6. Makino H, Nomura S, Maruyama H, Yokoyama T, Hirakata A, et al. (2019) Endoscopic application of chipping, over-scope clip (OTSC) and stenting for a fistula and anastomotic leakage of upper gastro-intestinal tract. Integr Cancer Sci Ther 6: 1000309

Copyright: (O2019 Miyamoto H. This is an open-access article distributed under the terms of the Creative Commons Attribution License, which permits unrestricted use, distribution, and reproduction in any medium, provided the original author and source are credited. 